\title{
Design and implement of high gain and low noise neural amplifier using compensation techniques
}

\author{
N.Manikandan, S.Muruganand, K.Karuppasamy \\ Department of electronics and Instrumentation, Bharathiar University, Coimbatore, Tamilnadu, India
}

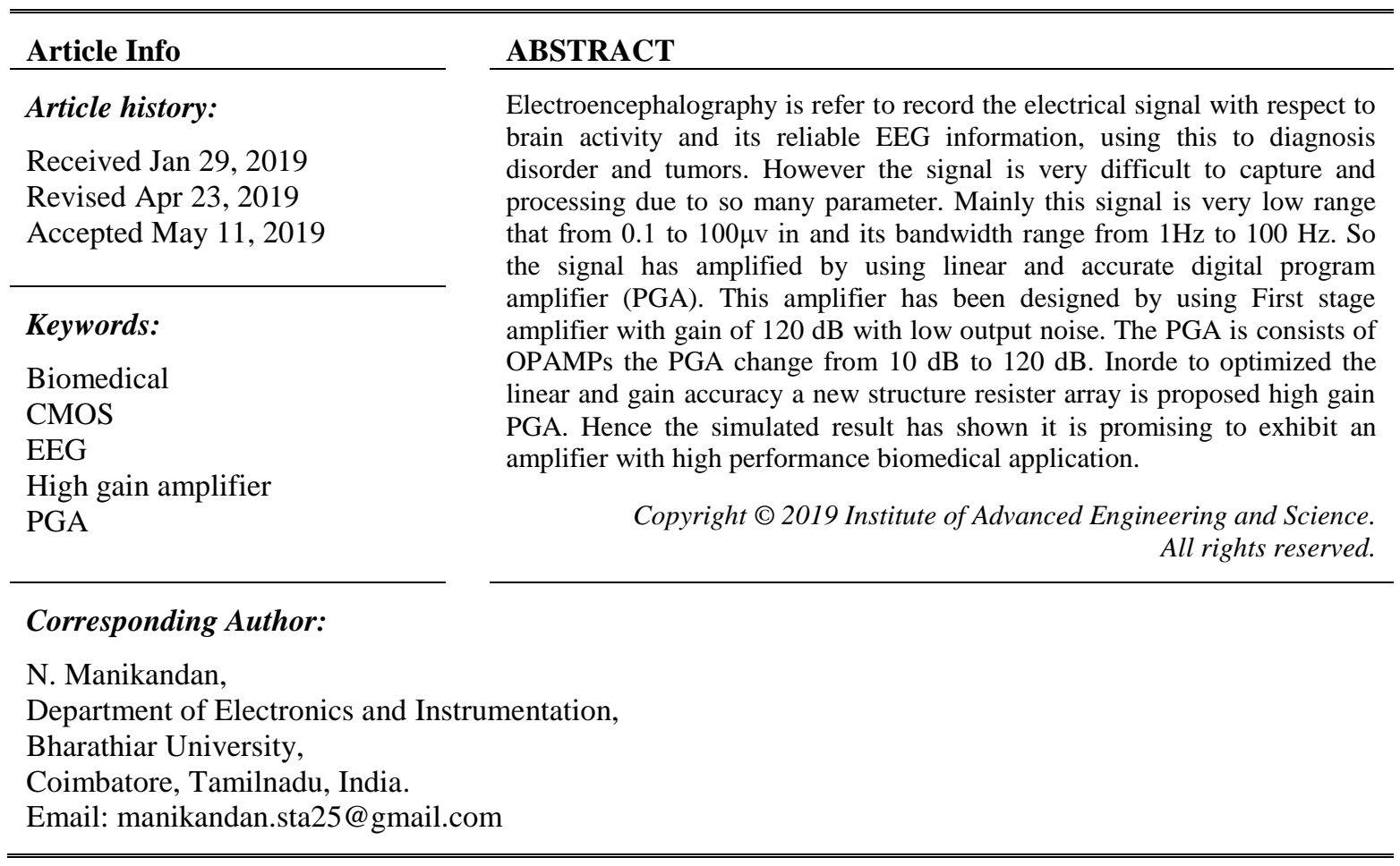

\section{INTRODUCTION}

The term medical instrument stood for simple hand-held instruments used by physicians for observing patients, examining organs, making simple measurements, or administering medication. These small instruments, such as stethoscopes, thermometers, tongue depressors, and a few surgical tools, typically fit into a physician's hand bag. Today's medical instruments are considerably more complicated and diverse, primarily because they incorporate electronic systems for sensing, transducing, manipulating, storing, and displaying data or information. Furthermore, medical specialists today request detailed and accurate measurements of a vast number of physiologic parameters for diagnosing illnesses and prescribe complicated procedures for treating these. As a result, the number of medical instruments and devices has grown from a few hundred a generation ago to more than 10,000 today, and the complexity of these instruments has grown at the same pace. The description of all these instruments and devices would fill an entire handbook by itself; however, due to the limited space assigned to this topic, only a selected number are described.

This section on medical instruments and devices neither replaces a textbook on this subject nor presents the material in a typical textbook manner $\mathrm{A}$ few traditional bioinstrumentation or electroencephalography have been omitted entirely because most textbooks on this subject give excellent introductions and reviews. Transducers, biosensors, and electrodes are covered in other sections of this Handbook. Thus this section provides an overview, albeit an incomplete one, of recent developments in the field of medical instruments and devices.

The ultimate goal is to implement a biopotential acquisition system that is comfortable and invisible to eye with long-term power autonomy, high signals. The aim is not only to increase the patients' quality of life but also to extend device applications to sports, entertainment, comfort monitoring, and so on. The biosignal used for these systems must have a high input impedance, a low output impedance, a limited 
bandwidth, and a low power consumption. Additionally it must have an adequate gain, a high power supply rejection ratio (PSRR), and common mode rejection ratio. The main circuit for any biopotential system is instrumentation amplifier. There is various ways to design instrumentation amplifier [2-6]. The primary thing for instrumentation amplifier is to have CMRR as high as possible to reduce the common mode signals in low amplitude and low frequency biopotential signal. The term instrumentation amplifier is properly used to describe a category of true differential input amplifiers that emphasize high common mode rejection (CMRR) and accuracy. Although both instrumentation amplifiers and difference amplifiers use op amps as basic architectural building blocks, they are distinctly different from their op amp cousins. In order to ease the dynamic range requirement of ADC, a PGA is placed in front of ADC. The decoder adjusts the output gain of PGA to an appropriate level which optimizes the performance of ADC. The circuit of PGA is realized in negative feedback i.e. a closed loop can achieve more precise gain setting and higher linearity. The gain control can be realized by varying the resistance of the input and feedback resistor array [7]. This paper is organized as follows. Part 2 describes the block diagram of proposed PGA. Part 3 presents the design consideration and circuit implementation.

\section{BASIC AMPLIFIER REQUIREMENTS}

The basic requirements that a biopotential amplifier has to satisfy are:

a) The physiological process to be monitored should not be influenced in any way by the amplifier

b) The measured signal should not be distorted

c) The amplifier should provide the best possible separation of signal and interferences

d) The amplifier has to offer protection of the patient from any hazard of electrical shock

e) The amplifier itself has to be protected against damages that might result from high input voltages as they occur during the application of defibrillators or electrosurgical instrumentation

The input signal to the amplifier consists of five components: the desired biopotential, undesired biopotentials, a power line interference signal of $60 \mathrm{~Hz}(50 \mathrm{~Hz}$ in some countries) and its harmonics, interference signals generated by the tissue/electrode interface, and noise. Proper design of the amplifier provides rejection of a large portion of the signal interferences.

The line frequency interference signal shows only very small differences in amplitude and phase between the two measuring electrodes, causing approximately the same potential at both inputs, and thus appears only between the inputs and ground and is called the common mode signal. Strong rejection of the common mode signal is one of the most important characteristics of a good biopotential amplifier. The common mode rejection ratio (or CMRR) of an amplifier is defined as the ratio of the differential mode gain over the common mode gain. As seen in Figure 1, the rejection of the common mode signal in a biopotential amplifier is both a function of the amplifier CMRR and the source impedances Z1 and Z2. For the ideal biopotential amplifier with $\mathrm{Z1=Z2}$ and infinite CMRR of the differential amplifier, the output voltage is the pure biological signal amplified by GD, the differential mode gain: Vout=GD. Vbiol. With finite CMRR, the common mode signal is not completely rejected, adding the interference term GD.Vc /CMRR to the output signal. Even in the case of an ideal differential amplifier with infinite CMRR, the common mode signal will not completely disappear unless the source impedances are equal. The common mode signal Vc causes currents to flow through $\mathrm{Z} 1$ and $\mathrm{Z} 2$. The related voltage drops show a difference if the source impedances are unequal, thus generating a differential signal at the amplifier input which, of course, is not rejected by the differential amplifier. With amplifier gain GD and input impedance Zin, the output voltage of the amplifier is:

$$
V_{\text {out }}=G_{d} V_{\text {bio }}+\frac{G_{D} V_{c}}{C M R R}+G_{d} V_{c}\left(1-\frac{Z_{\text {in }}}{Z_{\text {in }}+Z_{1}-Z_{2}}\right)
$$

The output of a real biopotential amplifier will always consist of the desired output component due to a differential biosignal, an undesired component due to incomplete rejection of common mode interference signals as a function of CMRR, and an undesired component due to source impedance unbalance allowing a small proportion of a common mode signal to appear as a differential signal to the amplifier. Since source impedance unbalances of 5,000 to $10,000 \Omega$, mainly caused by electrodes, are not uncommon, and sufficient rejection of line frequency interferences requires a minimum CMRR of $100 \mathrm{~dB}$, the input impedance of the amplifier should be at least $109 \Omega$ at $60 \mathrm{~Hz}$ to prevent source impedance unbalances from deteriorating the overall CMRR of the amplifier. State-of-the-art biopotential amplifiers provide a CMRR of 120 to $140 \mathrm{~dB}$.

A typical design of the various stages of a biopotential amplifier is shown in Figure 2. The electrodes which provide the transition between the ionic flow of currents in biological tissue and the electronic flow of current in the amplifier, represent a complex electrochemical system that is described 
elsewhere in this handbook. The electrodes determine to a large extent the composition of the measured signal. The preamplifier represents the most critical part of the amplifier itself since it sets the stage for the quality of the biosignal. With proper design, the preamplifier can eliminate, or at least minimize, most of the signals interfering with the measurement of biopotentials. In addition to electrode potentials and electromagnetic interferences, noise-generated by the amplifier and the connection between biological source and amplifier - has to be taken into account when designing the preamplifier. The total source resistance Rs, including the resistance of the biological source and all transition resistances between signal source and amplifier input, causes thermal voltage noise with a root mean square (rms) value:

$$
E_{r m s}=\sqrt{4 K T R B} w(\text { Volt })
$$

where $\mathrm{k}=$ Boltzmann constant, $\mathrm{T}=$ absolute temperature, Rs=resistance in $\Omega$, and $\mathrm{B}=$ bandwidth in $\mathrm{Hz}$. Additionally, there is the inherent amplifier noise. It consists of two frequency-dependent components, the internal voltage noise source $\mathrm{Vn}$ and the voltage drop across the source resistance Rs caused by an internal current noise generator in. The total input noise for the amplifier with a bandwidth of $B_{w}=f 2-f 1$ is calculated as the sum of its three independent components:

$$
E_{r m s}^{2}=\int_{f_{1}}^{f_{2}} V_{n}^{2}+R_{s}^{2} \int_{f_{1}}^{f_{2}} i_{n}^{2} d f+4 K T R_{s} B_{w}
$$

Hence eliminate all the noise signal high signal to noise ration thus require the use of very low niose amplifier is exhibition of voltage noise amplifier, then using high pass and low pass filter eliminate interference signal from the electrode potential and preamplifier potential offset and reduce noise amplifer amplitude band width since the biosignal amplitude disorder or attenuated by higher sharp-cutting linear phase filter have to be used. due to their smooth transfer function. Separation of biosignal and interference is in most cases incomplete due to the overlap of their spectra. The isolation stage serves the galvanic decoupling of the patient from the measuring equipment and provides safety from electrical hazards. This stage also prevents galvanic currents from deteriorating the signal-to-noise ratio especially by preventing ground loops. Various principles can be used to realize the isolation stage. Analog isolation amplifiers use either transformer, optical, or capacitive couplers to transmit the signal through the isolation barrier. Digital isolation amplifiers use a voltage/frequency converter to digitize the signal before it is transmitted easily by optical or inductive couplers to the output frequency/voltage converter. The most important characteristics of an isolation amplifier are low leakage current, isolation impedance, isolation voltage (or mode) rejection (IMR), and maximum safe isolation voltage.

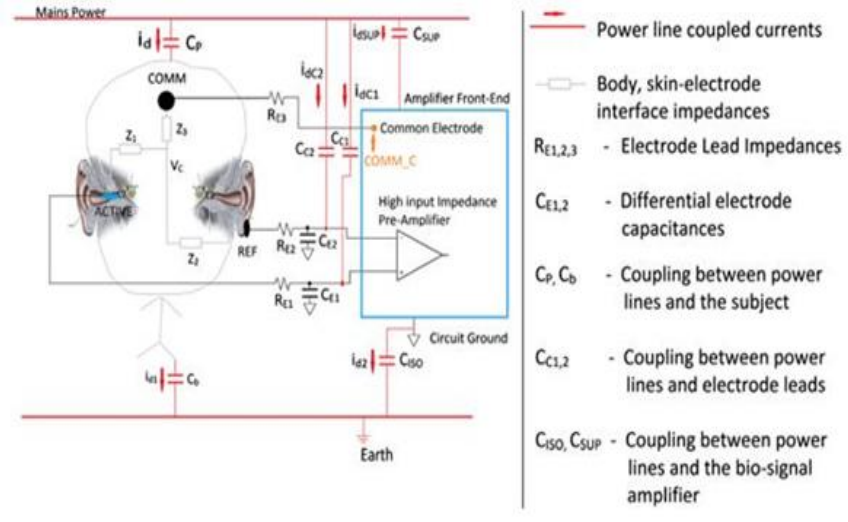

Figure 1. The neural amplifier connection

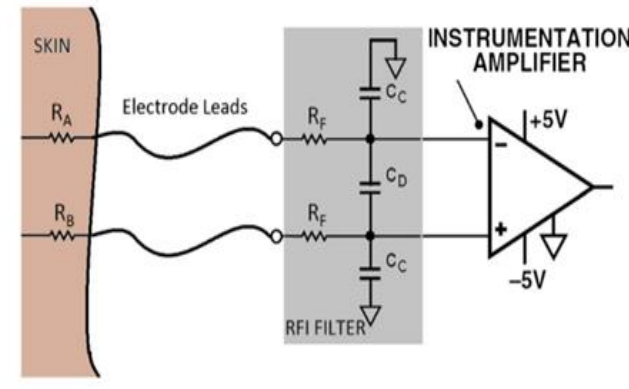

Figure 2. Impedance matching with amplifier

\section{ELECTRODE}

Potential difference at the cellular membrane may be in the range within $5 \mathrm{mV}$ and $100 \mathrm{mV}$. This potential difference is called the resting potential resting potential inside the cell is negative comparing to the environment resting potential of nerve and muscle cells is typically- $70 \mathrm{mV}$ to- $85 \mathrm{mV}$ the exchange charge 
carriers in electrical circuits, electrons are charge carriers-in the body, ions are charge carriers connects to the surface of the body (skin, mucous membranes) or on/in the organ inside the body model of the interface for better understanding of the interface electrode-tissue, Passive electrical characteristics of the skin-electrode interface strive to express by ideal electric components with intent parameters-Resistance-Capacity. This model can be used for measurement electrodes in limited frequency range. When a person is connected with electrodes for an EEG recording. The inputs to the amplifier withintrinsic impedances from electrodes/leads and skin, and power line displacement currents and their pathways. For our scenario CSUP is negligible.

The amplifier is battery powered. CE1 and CE2, are parasitic capacitances, that arise due to the shield conductor around the differential electrodes; however, PLI coupling from CC1 and CC2 to the inner conductors are reduced due to this shield. Byemploying active driven shields, the parasitic capacitances CE1 and CE2 will be minimized (detailed in part III B), thus displacement currents idC1, idC2, and idSUP are negligible. However, it must be noted that neglecting these displacementcurrents do not affect the common mode voltage (VC) derived in subsequent equations, since it is based on the total displacement current id 2 as derived in (3). Since displacement currents flowing in and out of the body must equate:

$$
\mathrm{i}_{\mathrm{d}}=\mathrm{i}_{\mathrm{d} 1}+\mathrm{i}_{\mathrm{d} 2}
$$

where id 2 is flowing from the body to the common (COMM) electrode. The current division between CISO and $\mathrm{Cb}$ is used to express id 2 as follows:

$$
i_{d 2}=\frac{C_{I S O}}{C_{i s o}+C_{d}} i_{d}
$$

Then the common mode voltage VC is where ZRLD is the impedance between the VC node and circuit ground. If the COMM electrode was connected to circuit ground, then:

$$
\mathrm{Z}_{\mathrm{RLD}}=\frac{Z_{3+R_{E 3}}}{G+1}
$$

The COMM electrode driven by the RLD circuit. The RLD circuit consists of an inverting amplifier with gain with a corner frequency set at $1.5 \mathrm{kHz}$. The RLD circuit is driven by the signal common to both the differential electrodes (known as the common mode voltage). By driving the COMM electrode with an inverted VC with gain $(\mathrm{G}+1)$, the effective impedance ZRLD simplifies to the final Vc is;

$$
i_{d 2}=\frac{C_{I S O}}{C_{i s o}+C_{d}} i_{d} \cdot Z_{3+R_{E 3}}
$$

In commonly used bio-signal measurements, such as ECG/EEG/EMG, bandwidth reduction from the RFI filter due to large input impedances is negligible since the required signal bandwidth is $<1 \mathrm{kHz}$. In contrast, the signal bandwidth of EVestG recordings spans up to $10 \mathrm{kHz}$, hence will be affected by bandwidth reduction.Secondly, with the traditional method, $\mathrm{CD}$ and $\mathrm{CC}$ capacitors need to be in the pF range to maintain high impedance at the electrode terminals, therefore large resistors $(>5 \mathrm{k} \Omega)$ are required to realize the desired corner frequencies.

The thermal noise of $\sim 9 \mathrm{nV} \mathrm{pHz}$ from a $5 \mathrm{k} \Omega$ resistor is clearly unacceptable for this work, since the stringent low noise requirements wouldn't be achievable if the traditional RFI filter network was employed. With the aid of the proposed RFI filter topology the following were achieved: Large mismatched input impedances will have no effect on the RFI corner frequencies. It is possible to set RF at $56 \Omega$, that has an insignificantly small thermal noise $0: 96 \mathrm{nVpHz}$ contribution, since there is no strict restriction on the values $\mathrm{CD}$ and $\mathrm{CC}$ to satisfy the required $\mathrm{RC}$ value. The capacitances $\mathrm{CD}$ and $\mathrm{CC}$ were therefore set at $1 \mathrm{nF}$ and 5.6 $\mathrm{nF}$ respectively to obtain a $200 \mathrm{kHz}$ cut off frequency, while accounting for $\sim 50 \Omega$ output impedance on each side of the paralleled high impedance buffers.

\section{DESIGNED OF AMPLIFIER}

The design of a high gain, low power, general purpose op amp with the structural simplicity of the classical Widlar architecture. The proposed op amp structure applies composite cascode connections in both the input stage and the second stage to achieve a gain of around $120 \mathrm{~dB}$ with low power consumption. The op amp employs the traditional two gain stages followed by a near unity gain buffer stage. With only two gain stages, simple Miller compensation with a capacitor as small as $3.5 \mathrm{pF}$ can be used. This approach overcomes some limitations of conventional CMOS cascodes by enhancing the gain without using additional bias 
circuits and requires only a small bias headroom voltage. A larger output voltage swing is then available using composite cascode amplifier stages than with conventional cascodes. As discussed in the former chapter, low power op amp can be designed by operating the MOS transistors in the subthreshold or weak inversion region. While the composite cascode provides high output impedance at a low bias current while setting some devices in the subthreshold region along with the other active region transistors, low power dissipation and high gain can be achieved at the same time. Figure 3 shows second stage cascade amplifier.

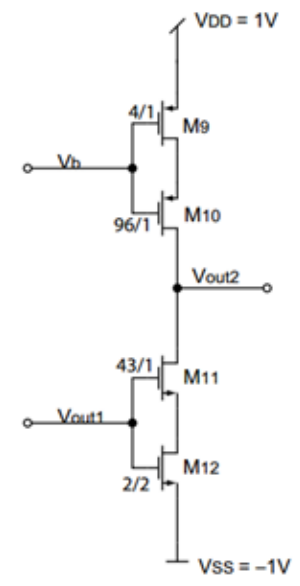

Figure 3. Second stage cascade amplifier

The cascade of amplifier which is amp the volt signal in the range from- 1 to $0.7 \mathrm{v}$ the linear range is obviously the range of interest in an op amp. It denotes the voltage range over which the two inputs can be driven together even though only one input terminal of the op amp is actually connected with the input signal without causing common mode distortion. The proposed compact high-gain op amp is laid out $0.25 \mu \mathrm{m}$ process and fabricated. The slew rate and $1 \%$ settling time have been measured when the op amp is in the unity-gain feedback configuration with a $0.5 \mathrm{~V}$ step input. More than half of the power consumption is due to the output source follower. The input stage and second stage dissipated little power because of the low current weak inversion operating region. Using simple Miller capacitor compensation, $\mathrm{Cc}$ is required to be only $3.5 \mathrm{pF}$. The micrograph of the opamp is the area of the amplifier is about $0.05 \mathrm{~mm}^{2}$ and it could be reduced more if necessary. The simulated gain of this opamp is $120 \mathrm{~dB}$. Schematic of cascade amplifier is as shown in Figure 4. Graphic of cascade amplifier is as shown in Figure 5.

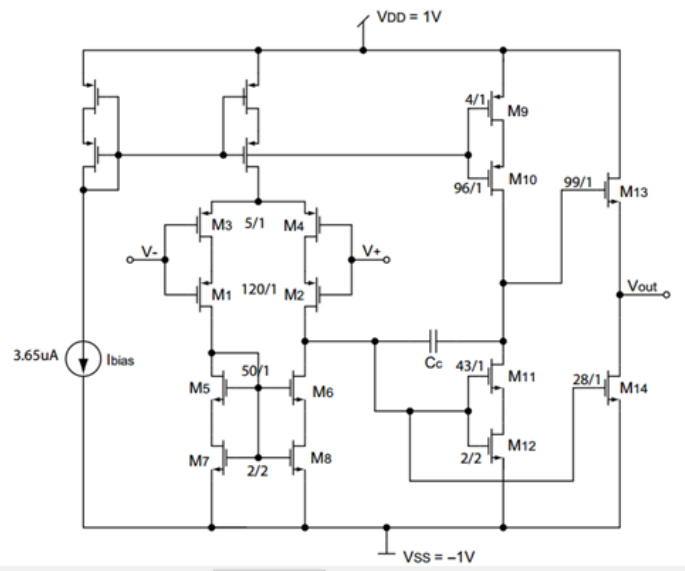

Figure 4. Schematic of cascade amplifier

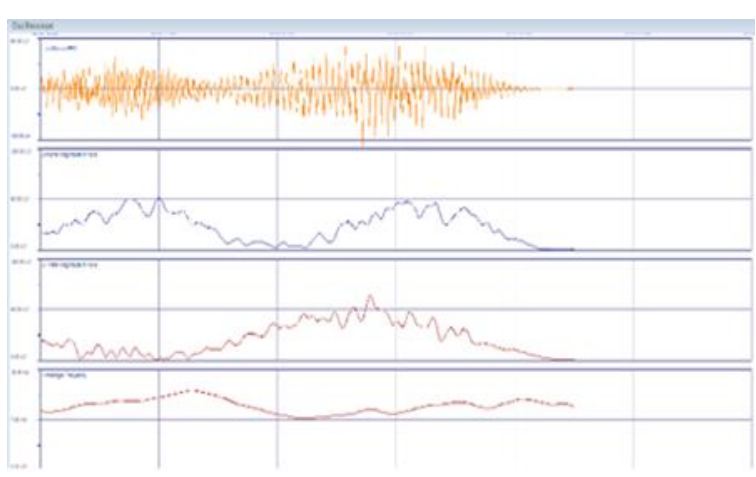

Figure 5. Graphic of cascade amplifier 


\section{CONCLUSION}

This work reports a low power, high gain op amp design in a $0.25 \mu \mathrm{m}$ CMOS process using the composite cascode connection. The proposed op amp produces an open loop gain above one million. The design circuit is tested. It has shows a gain of about $117 \mathrm{~dB}$. In addition, the composite cascode amplifier requires a compensation capacitor of only $3.5 \mathrm{pF}$ which allows a very small op amp cell. This design is intended for applications where simplicity of layout, small cell size, and low power are important. The power supply voltage can be further reduced from 2 volt to 1.5 volt due to the freedom in biasing the subthreshold devices.

\section{REFERENCES}

[1] H. Lee, K. N. Leung, and P. Mok, "A dual-path bandwidth extension amplifier topology with dual-loop parallel compensation,” IEEE J. Solid-State Circuits, vol. 38, no. 10, pp. 1739-1744, Oct. 2003.

[2] B. Shem-Tov, M. ozak, and E. Friedman, "A high-speed CMOS op-amp design technique using negative miller capacitance," Proceedings of the 2004 11th IEEE International Conference on Electronics, Circuits and Systems, pp. 623-626, Dec.2004.

[3] E. Waterhouse,-New horizons in ambulatory EEG monitoring, IEEE Eng. Med. Biol. Mag., vol. 22, no. 3, pp. 74-80, May/Jun. 2003.

[4] N.Manikandan" International Journal of Electronics and Electrical Engineering Vol. 3, No. 4, August 2015

[5] C Enz,-Analysis of the low-frequency noise reduction by autozero technique,\| Electron. Lett., vol. 20, pp. 959960, Nov

[6] Szynowski,-CMRR analysis of instrumentation amplifiers,\|Electron. Lerr., vol. 19, no. 14, pp. 547-549, 1983

[7] J. Szynowski,-CMRR analysis of instrumentation amplifiers, IElectron. Lerr., vol. 19, no. 14, pp. 547-549, 1983

[8] PedramMohseni, Student Member, IEEE, and Khalil Najafi, Fellow, IEEE(2004), "A Fully Integrated Neural Recording Amplifier With DC Input Stabilization “,IEEE transactions on biomedical engineering, vol. 51, no. 5, may 2004 ,

[9] K. D. Wise(1969), "A multi-channel microprobe for bio potential recording," Ph.D. dissertation, Stanford Univ., Stanford, CA, 1969

[10] P. M. Van Peteghem, I. Verbauwhede, and W. M. C. Sansen(1985), "Micro-power high-performance SC building block for integrated low-level signal processing,"IEEE J. Solid-State Circuits, vol. SC-20, pp. 837-844, Aug. 1985.

[11] Hwang-Cherng Chow and Jia-Yu Wang(2007), "High CMRR instrumentation amplifier for biomedical applications “, Department and Graduate Institute of Electronics Engineering Chang Gung University, Tao-Yuan 333, Taiwan, ROC,2007 IEEE

[12] E. L. Douglas , D.F. Lovely and D.M. Luke(2004), "A Low-Voltage Current-mode Instrumentation Amplifier Designed in a 0.18-Micron CMOS Technology," in Proc. IEEE CCECE, pp. 1777-1780, 2004.

[13] R. Pallas-Areny and J. G. Webster(1991), "Common Mode Rejection Ratio for Cascoded Differential Amplifier Stages," IEEE transaction on instrumentation and measurement, vol. 40, no. 4, pp. 677-681, 1991

[14] Maryam Shojaei-Baghini, Rakesh K. Lal, Dinesh K. Sharma(2005), "A Low-Power and Compact Analog CMOS Processing Chip for Portable ECG Recorders",2005,ISSN-0-78 03-9162-4, IEEE

[15] A. Subasi, E. Ercelebi, Classification of EEG signals using neural network and logistic regression, Comput. Methods Prog. Biomed. 78 (2005) 87-99.

[16] I. Guler, E.D. Ubeyli, Multiclass support vector machines for EEG-signals classification, IEEE Trans. Inf. Technol. Biomed. 11 (2) (2007) 117-126.

[17] J. Stastny, P. Sovka, A. Stancak, EEG signal classification:introduction to the problem, Radioengineering 12 (3) (2003) 51-55.

[18] S. Poyhonen, A. Arkkio, P. Jover, H. Hyotyniemi, Couplingpairwise support vector machines for fault classification, Control Eng. Pract. 13 (2005) 759-769.

[19] F. Benimeli, K. Sharman, Electroencephalogram signal classification for brain computer interfaces using wavelets and support vector machines, in: ESAN'2007 Proceedings European Symposium on Artificial Neural Networks, Bruges, Belgium, 25-27 April, 2007, pp. 361-366. 